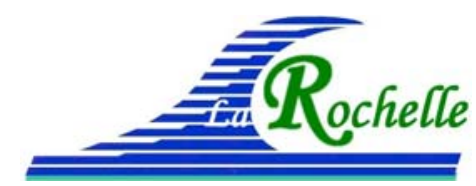

XVìmes Journées Nationales Génie Côtier - Génie Civil

La Rochelle, 29 au 31 mai 2018

DOI:10.5150/jngcgc.2018.060 @ Editions Paralia CFL

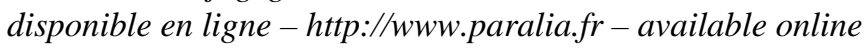

\title{
Le raccordement des EMR avec un procédé novateur à faible impact environnemental : Le POSÉIDON ${ }^{\circledR}$
}

\author{
Philippe DHERVILLY ${ }^{1}$, Daniel RIVARD ${ }^{1}$, \\ Daniel LEVACHER ${ }^{2}$, Nacer ALLOUN ${ }^{1,2}$
}

1. Groupe Marais, 1 rue Pierre et Marie Curie, 49430 Durtal, France. p.dhervilly@samarais.com

2. Université de Normandie, Unicaen, UMR 6143 CNRS - M2C, 24 rue des Tilleuls, 14000 Caen, France.

daniel.levacher@unicaen.fr

\section{Résumé :}

Le procédé POSÉIDON ${ }^{\circledR}$ est composé d'une ensouilleuse étanche équipée d'une roue ou d'un soc de tranchage. Cette unité à propulsion électrique, pilotée depuis la surface par un poste de commande placé sur une barge. Il permet d'exécuter une profondeur de coupe jusqu'à $1300 \mathrm{~mm}$ et une largeur de coupe jusqu'à $250 \mathrm{~mm}$ afin de réaliser la pose mécanisée simultanée de câbles ou de fourreaux stockés sur la barge à la surface de l'eau. Ce procédé occasionne peu de gêne sur le trafic maritime ou fluvial et respecte l'environnement avec la reconstruction naturelle du fond marin ou de la rivière.

Les avantages techniques et environnementaux pour la pose des câbles reliant les EMR au continent sont décrits ci-après.

\section{Mots-clés :}

Génie côtier, EMR, Tranchées sous-marines, Travaux maritimes, Ecosystèmes côtiers, Trancheuse

\begin{abstract}
:
POSÉIDON $^{\circledR}$ process has very low impact on the aquatic environment that makes it a unique technology particularly adapted to the shallow depths characterizing the locations of EMR. It allows cutting depths up to $1300 \mathrm{~mm}$, cutting width up to $250 \mathrm{~mm}$ and perform the simultaneous mechanical installation of cables or sleeves stored on the towed barge on the surface of the water. The advantages of the process are based on an intelligent use with work causing little inconvenience on the traffic, on the respect of the environment with the natural reconstruction of the bed of the river or the river and that the passage of networks does not generate any impact on the aquatic ecosystem. The machine, its process and advantages in laying submarine cables and some applications are presented in the paper.
\end{abstract}

Keywords: Coastal engineering, RME, Underwater trenches, Marine works, Coastal ecosystems, Slicing machine. 


\section{Thème 5 - Énergies et ressources marines}

\section{Introduction}

Les énergies renouvelables constituent une voie d'avenir. Elles contribueront non seulement à l'indispensable lutte contre le changement climatique, mais aussi au développement des énergies nouvelles. Parmi ces formes d'énergies, celles des mers et des océans (EMR). La France s'est déjà fixé l'ambition de figurer parmi les leaders de ce secteur en forte croissance à l'échelle européenne avec un objectif de $6000 \mathrm{MW}$ d'énergies marines renouvelables installés en 2020 (syndicat des énergies renouvelables).

Situés au large des côtes, les parcs éoliens ne peuvent être directement raccordés à des stations de conversion ou transformateurs et nécessite la pose de kilomètres de câbles qui doivent êtres enfouis dans les fonds pour des raisons de sécurité.

Le procédé POSÉIDON, ${ }^{\circledR}$ porté par le Groupe MARAIS, développe et qualifie un concept totalement innovant basé sur l'enfouissement de réseaux de télécommunication en milieu marin. Ces connexions électriques sous-marines permettent de transmettre les fortes puissances produites par les systèmes d'énergies renouvelables en mer, tout en réduisant le coût et l'impact environnemental sur le milieu aquatique.

\section{Les caractéristiques du raccordement}

Le raccordement des parcs éoliens par une liaison sous-marine, jusqu'à un point d'atterrage situé sur le littoral, nécessite deux liaisons, sous-marine et terrestre.

Arrivés sur la côte, les câbles sous-marins sont reliés au réseau terrestre existant ce qui n'est pas sans poser un certain nombre de problèmes.

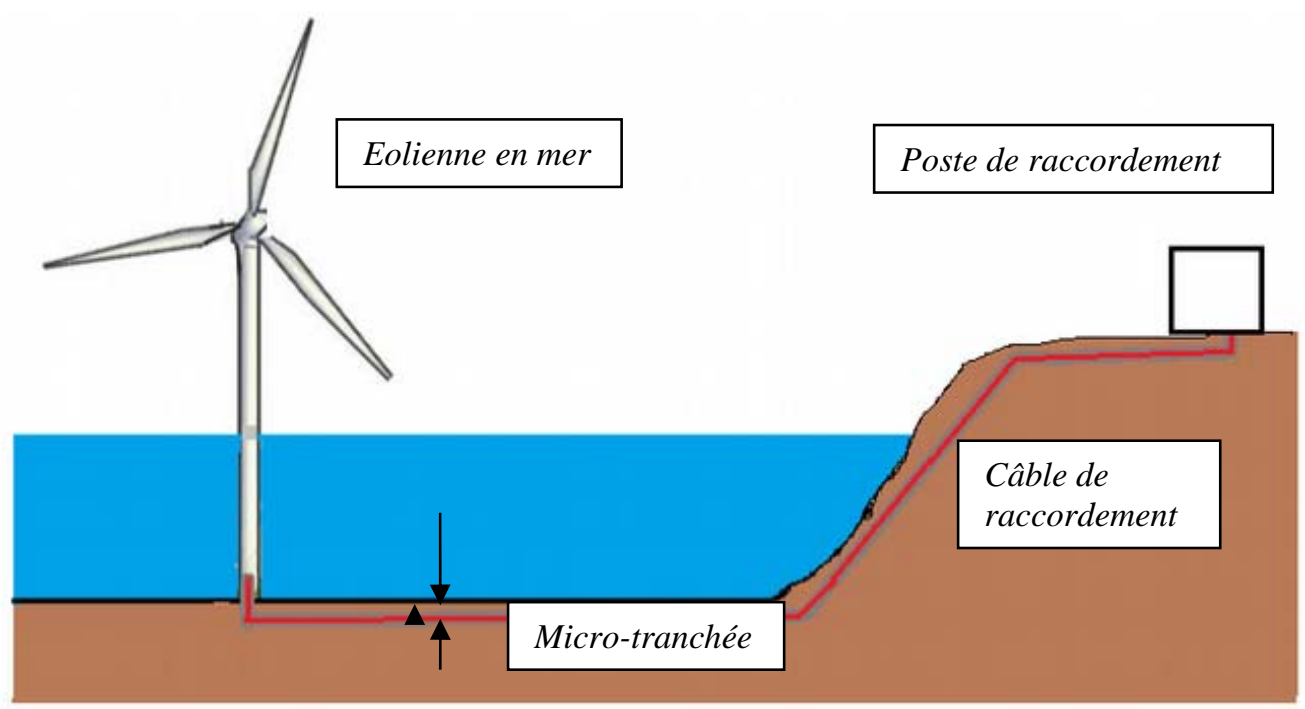

Figure 1. Raccordement des éoliennes avec des câbles mécanisés. 


\section{XVèmes Journées Nationales Génie Côtier - Génie Civil \\ La Rochelle, 29 au 31 mai 2018}

\section{Pose de réseaux en milieu marin}

Le procédé POSÉIDON ${ }^{\circledR}$ est un concept basé sur l'enfouissement de réseaux de télécommunication en milieu marin. En surface une barge automotrice porte les matériels nécessaires à l'ouverture de la tranchée dans le lit sous- marin. Il permet :

- Une profondeur de coupe jusqu'à $1110 \mathrm{~mm}$ pour la roue de tranchage et $1300 \mathrm{~mm}$ pour le soc vibrant ;

- Une largeur de coupe jusqu'à $250 \mathrm{~mm}$ pour la roue de tranchage et $90 \mathrm{~mm}$ pour le soc vibrant ;

- La pose mécanisée simultanée de câbles ou de fourreaux stockés sur la barge.

Les tranchées réalisées ont une profondeur qui varie généralement entre $0,50 \mathrm{~m}$ et 1,30 $\mathrm{m}$ et une largeur comprise entre 0,25 et $0,30 \mathrm{~m}$.

Le remblayage de ces tranchées se fait d'une manière naturelle, pour des raisons liées à la nature des sédiments marins, les tranchées ont tendance à remblayer au fur à mesure de la pose des câbles.

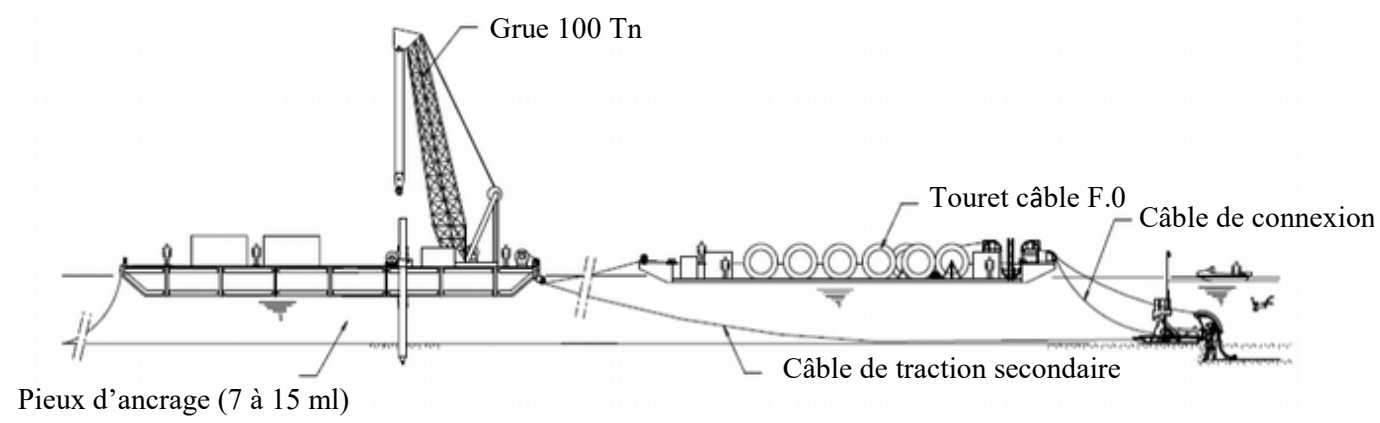

Figure 2. Schéma de procédé POSÉIDON ${ }^{\circledR}$.

Tableau 1. Caractéristiques de l'outil de coupe.

\begin{tabular}{lll}
\hline Version & Largeur & Profondeur \\
\hline Soc vibrant & $100 \mathrm{~mm}$ & $1300 \mathrm{~mm}$ \\
Roue de tranchage & $250 \mathrm{~mm}$ & $1100 \mathrm{~mm}$ \\
\hline
\end{tabular}

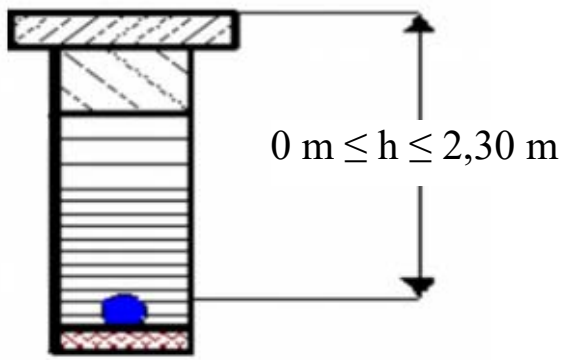

$0,25 \stackrel{\longmapsto}{\mathrm{m}<1 \leq 0,35 \mathrm{~m}}$

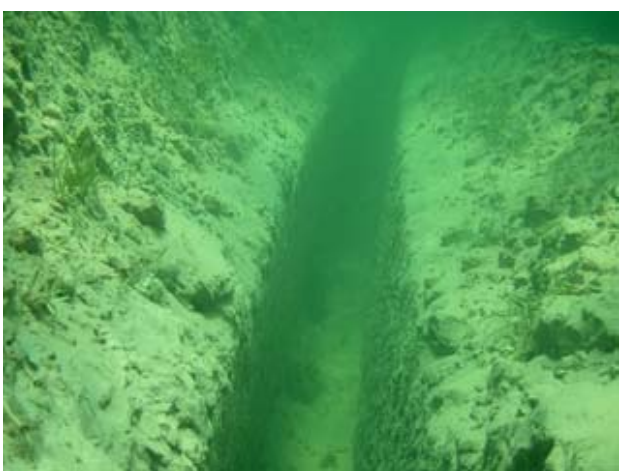

Figure 3. a) Dimensions des micro-tranchées. b)Micro--tranchée sous-marine. 


\section{Thème 5 - Énergies et ressources marines}

\section{Utilité d'un tel procédé}

Ces travaux occasionnent peu de gêne sur le trafic, et respectent l'environnement avec la reconstruction naturelle du lit fluviatile ou marin. Enfin, le passage de réseaux n'occasionne aucune mauvaise influence sur l'écosystème. Il peut être réalisé jusqu'à $2500 \mathrm{ml} /$ jour de tranchées pour la pose des réseaux.

\section{Trancheuses spécifiques pour la pose mécanisée de réseaux en milieu marin}

\subsection{Machine d'ensouillage POSEIDON® 1}

Cette machine est montée sur un porteur à chenilles destiné à l'ensouillage de câbles sous-marins. Conçue et fabriquée par MARAIS technologies, elle est opérée depuis la surface. Selon le type de sol, elle peut être équipée de différents outils : roue de tranchage, chaîne à picots.

Tableau 2. Caractéristiques principales de la machine

\begin{tabular}{|c|c|c|c|c|c|c|}
\hline $\begin{array}{l}\text { Longueur } \\
\text { H.T. (m) }\end{array}$ & $\begin{array}{l}\text { Largeur } \\
\text { H.T. (m) }\end{array}$ & $\begin{array}{l}\text { Hauteur } \\
\text { H.T. (m) }\end{array}$ & $\begin{array}{l}\text { Masse dans } \\
\text { l'air (t) }\end{array}$ & $\begin{array}{l}\text { Masse dans l'eau } \\
(t)\end{array}$ & $\begin{array}{l}\text { Puissance } \\
\text { (cv) }\end{array}$ & $\begin{array}{l}\text { Profondeur } \\
\text { d'immersion (m) }\end{array}$ \\
\hline 12,50 & 5,62 & 2,52 & 27 & 20 & 450 & 120 \\
\hline
\end{tabular}

Elle est conçue dans l'objectif de réalisation de tranchées et de pose mécanisée simultanées pour le déploiement de réseaux (télécoms, électriques, eau, assainissement, gaz, etc) en milieu marin, ou en milieu estuarien.

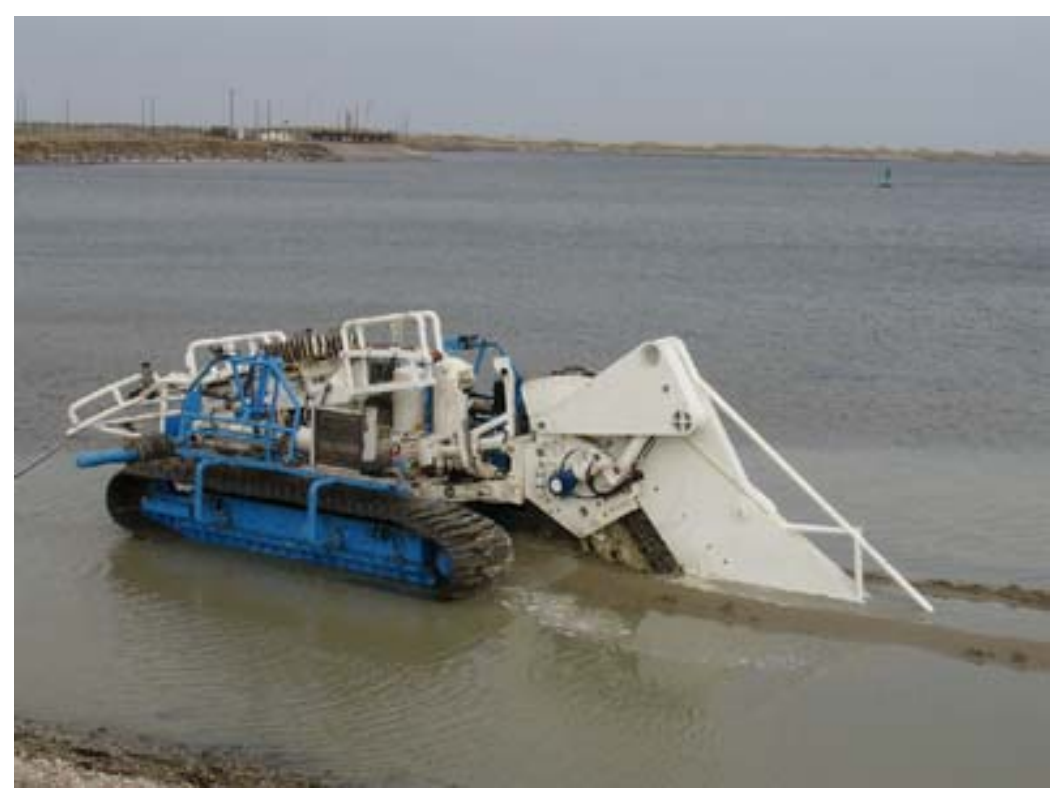

Figure 4. Machine d'ensouillage POSEIDON® 1 


\section{XVèmes Journées Nationales Génie Côtier - Génie Civil \\ La Rochelle, 29 au 31 mai 2018}

\subsection{Machine d'ensouillage POSEIDON® 2}

La machine est composée d'un porteur sur chenilles destiné à l'ensouillage de câbles sous-marins. Conçue et fabriquée par MARAIS, cette machine permet l'enfouissement sans assistance de plongeurs. Opérée depuis la surface, elle peut être équipée de différents outils selon le type de sol: roue de tranchage avec jetting et/ou éducteur.

Tableau 3. Caractéristiques principales de la machine et des tranchées réalisées.

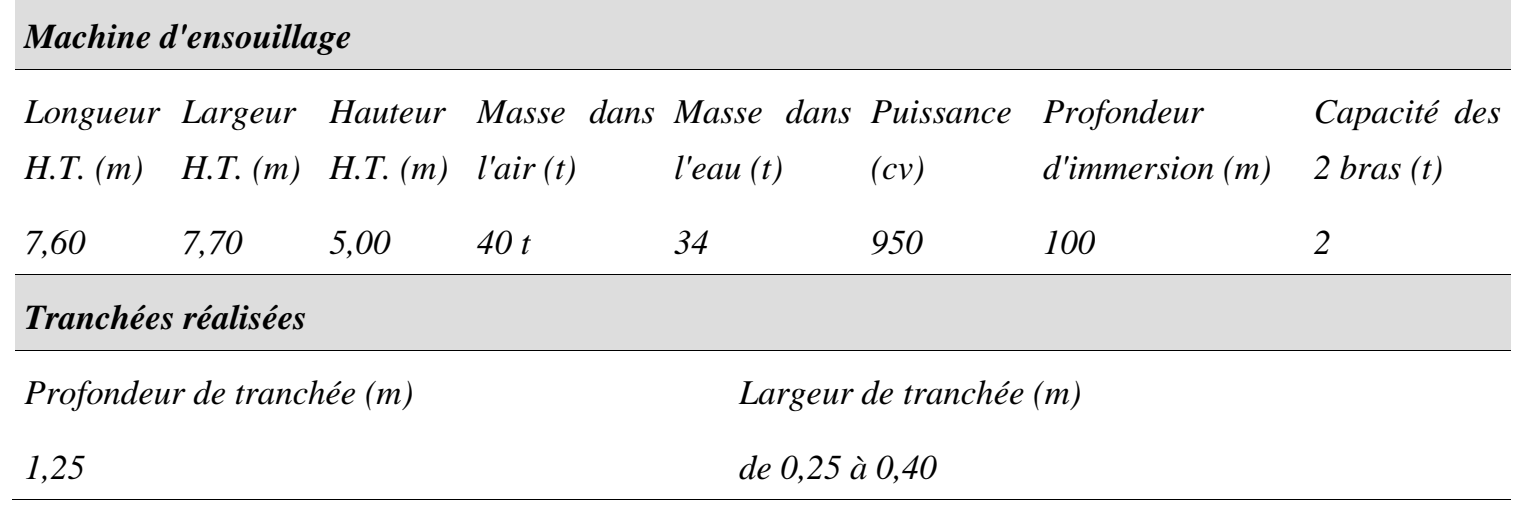

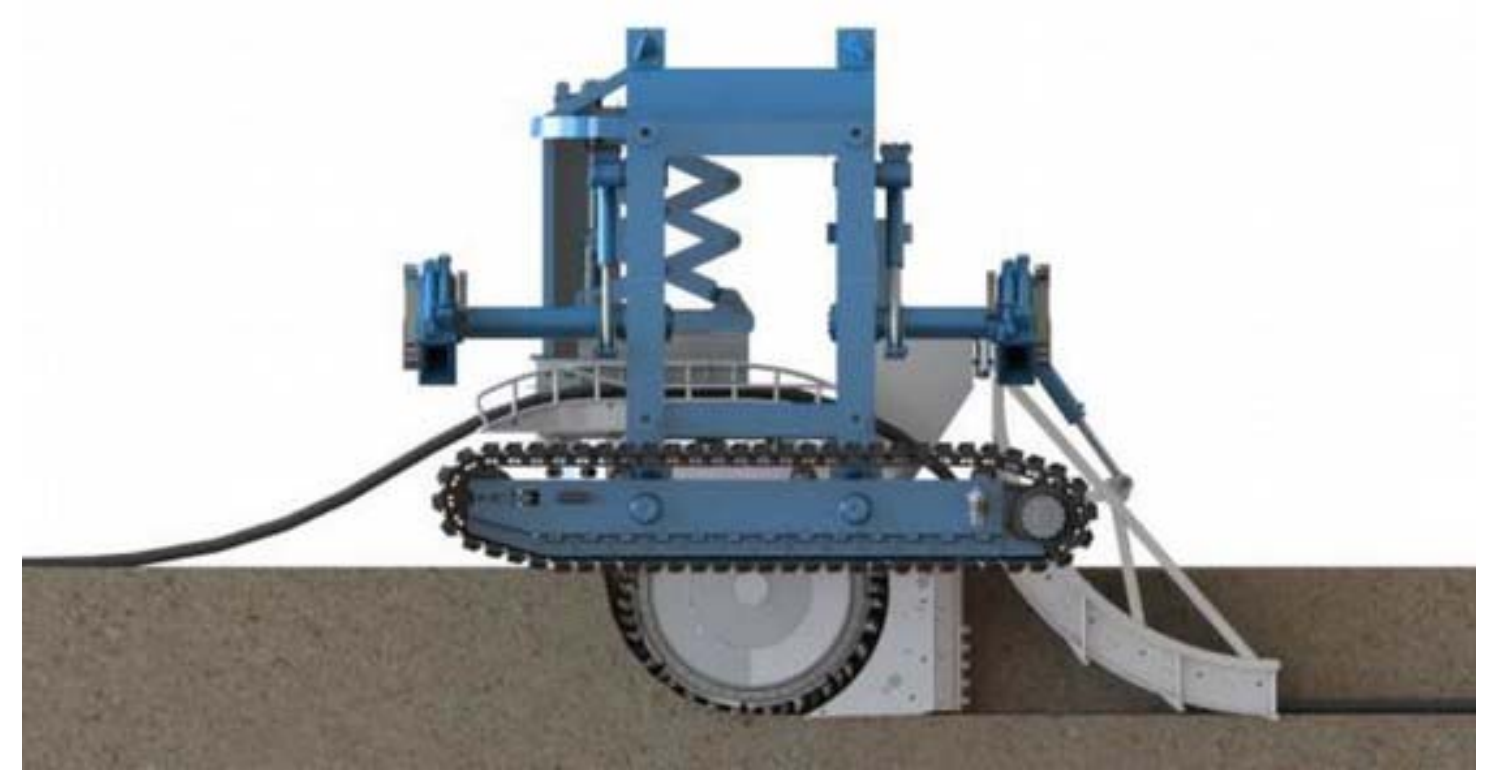

Figure 5. Machine d'ensouillage POSEIDON®.

\section{6. Étude géotechnique des substrats des tranchées}

Les caractéristiques des fonds marins influencent le choix des méthodes de protection des câbles qui seront utilisées, et la profondeur des tranchées qui seront réalisées. Les 


\section{Thème 5 - Énergies et ressources marines}

conditions particulières d'implantation de ces réseaux seront conçus pour minimiser les risques liés à la navigation et les travaux maritimes.

De par sa conception POSEIDON ${ }^{\circledR}$ réduit de façon significative les marqueurs environnementaux. Il supprime l'apport de matériaux depuis la surface pour le rebouchage et limite la remise en suspension des sédiments.

L'utilisation d'énergie électrique pour son alimentation réduit également les risques de pollution liés aux pertes d'huile. L'emploi de produits 100\% biodégradables est un atout supplémentaire.

\section{Conclusion}

Les champs éoliens en mer sont, en France, à l'aube d'un très fort développement et représentent des défis technologiques et environnementaux majeurs. Afin de mieux faire accepter ces nouveaux obstacles à la vie maritime côtière, nous devons imaginer de nouveaux systèmes d'assemblage de ces parcs, totalement respectueux de l'environnement. POSEIDON ${ }^{\circledR}$ répond parfaitement aux problématiques de raccordement des fermes éoliennes aux réseaux terrestres.

Cette machine produit un très faible impact sur les fonds marins et, de par sa conception, n'émet aucun rejet dans le milieu naturel.

Dans ce cadre la société Groupe MARAIS a réalisé au cours des trois dernières années plus de $6575 \mathrm{~km}$ de réseaux, dont $25 \%$ sont des travaux de connexions inter-éoliennes. Ses principaux clients dans ce secteur sont : EDF, BOUYGUES, VINCI, SPIE, INEO, CEGELEC, SOBECA, NGE etc.

\section{Références bibliographiques}

EDF (2013). Projet de parc éolien en mer au large de Courseulles-sur-Mer, pp 5-7.

GICAN (2016). L'industrie maritime française s'engage pour les énergies marines renouvelables, pp 10-11.

GROUPE MARAIS (2016). Enfouissement de câbles et fourreaux sur voies navigables. RIE (2015). Marines renouvelables les enjeux des énergies, pp 12-17.

RIE (2017). Raccordement électrique du parc éolien en mer, pp 3-5.

TRAVOCEAN (2009). Machine d'ensouillage (outils optionnels : pompe de dragage, jetting).

\section{Références sitographiques}

http://www.samarais.com/fr/groupe/organisation_du_groupe/chantiers_realises.html 